\title{
Temperatures effects on cascaded Mach-Zehnder interferometer structures
}

\author{
Ary Syahriar, Rahmat Alamtaha, Zulkifli Alamtaha, Putri Wulandari \\ Department of Electrical Engineering, Faculty of Science and Engineering, University of Al-Azhar Indonesia, Indonesia
}

\begin{tabular}{l}
\hline \hline Article Info \\
\hline Article history: \\
Received Sep 2, 2019 \\
Revised Dec 29, 2019 \\
Accepted Feb 20, 2020 \\
\hline
\end{tabular}

\section{Keywords:}

Cascaded MZI

Spectral response

Temperatures effects

Transfer matrix

Wavelength shift

\begin{abstract}
To increase bandwidth and number of channels per fiber for more than one wavelength in the same fiber the dense wavelength division multiplexing (DWDM) technology has been utilized. One of the devices that are important in DWDM is an optical interleaver. This paper discussed the effects of temperatures in the DWDM interleaver by using the Mach-Zehnder interferometer (MZI) structures which is arranged in two-stage cascaded MZI and the three-stage cascaded MZI geometries. The main consequences of increase temperature inside the fiber optics are the change of effective refractive index in the material of silica fiber due to the thermo-optics effects. In our analysis we have used the transfer matrix method to investigate the wavelength dependence of output power to the temperatures changes that varies from $30^{\circ} \mathrm{C}$ to $430^{\circ} \mathrm{C}$. In the calculation we have used the C-Band range wavelength which is around 1530 to $1565 \mathrm{~nm}$. It has been shown that the change of temperatures may shift the wavelength inside the MZI output power in linear manners. These effects may be used to tune wavelength transmission inside the MZI structures to suit the ITU-T defined grid.
\end{abstract}

This is an open access article under the CC BY-SA license.

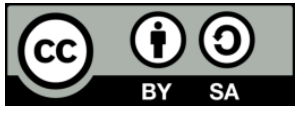

\section{Corresponding Author:}

Ary Syahriar,

Department of Electrical Engineering,

University al Azhar Indonesia,

Kompleks Masjid Agung al Azhar, Jl. Sisingamangaraja, Jakarta Selatan, Indonesia.

Email: ary@uai.ac.id

\section{INTRODUCTION}

Recent explosion on the Internet growth has put pressure on optical fiber network to expand its channels. To fulfill the requirements on increasing information carrying capacity of optical fiber communication systems the dense wavelength division multiplexing (DWDM) technology might be utilized. In DWDM system a number of devices have been used which include laser sources with different optical wavelength, multiplexer/demultiplexer to combine and spread the signals at the transmitter and receiver side. DWDM technology also offers the flexibility to enhance network capacity without adding more fiber optics into the existing systems. Therefore, the DWDM can further increase the channel data rates or increasing the number of multiplexed channels [1].

In principle, DWDM devices that can be used as a set of optical channels, each of which uses a different wavelength [2]. Interleavers are becoming an interesting research focus on the DWD systems because it can design to provide new effective solutions. An optical interleaver is a device that combines two DWDM channels into multiple signals stream in interleaving ways or vice versa [3]. Interleaver light signals work similar to comb filters, it can split one signal into two or more signals with greater intervals between them [4]. A method is often used to design filters in optical fiber is the Interleaver method based on Mach-Zehnder interferometer (MZI) structure [5]. 
The MZI has been developed to act as a switch (in its balanced form) and as a multiplexer or demultiplexer (in its unbalanced form). The MZI consists of two directional couplers or Y-junctions, connected by linking fiber optics. Introducing a phase delay to one linking guide via the thermo-optic effect enables the MZI to be used as a switching device. Based on the number of couplers, the configuration of MZI is divided into several types, namely single MZI consists of two couplers and cascaded MZI use more than two couplers [5-7]. The characteristics and the design of MZI have been the focus of discussion in developing sensitive filters and sensors $[8,9]$.

In this paper, cascaded MZI structure is used as optical interleaver with one input, namely on port $1[9,10]$. In general, interleaver devices can operate well in a certain temperature range. However, outside the predetermined temperature range, the signal transmission characteristics on the interleaver device will change $[11,12]$. Based on this problem, we simulated the effect of heating two-stage and three-stage successive MZI with the range of temperature from $30^{\circ} \mathrm{C}$ to $430^{\circ} \mathrm{C}$.

\section{RESEARCH METHOD}

\subsection{Temperatures effects on silica fibers}

The thermo-optics (TO) effect is refractive index changes inside the fiber optics as the temperature rises $[12,13]$. Usually, the TO phase shifters are comprises of a thin film heater placed on the fiber optics. When the heater is on the material refractive index will change, for example heating a $10 \mathrm{~mm}$ long guide at temperature of $15.23^{\circ} \mathrm{C}$, will create a $\pi$ radians phase shift at $1.523 \mu \mathrm{m}$ wavelength [8]. The phase shift values and time to heat the materials depend on the cladding thickness, thermal characteristics, and substrate material used [9].

At this time, there has been a new research to determine the new relation in equation and thermal-optical coefficients in silica fibers at temperatures ranges of $0-1200^{\circ} \mathrm{C}$. The relation also includes second order coefficients of thermo-optics as follow [14].

$$
n(T)=n_{0}+\alpha_{n} T+\beta_{n} T^{2}
$$

where $n(T)$ is the effective refractive index as a function of temperature. $\alpha_{n}$ and $\beta_{n}$ are first-order and second-order thermo-optic coefficients respectively. In this calculation we adopt the values as $\alpha_{n}=1.090 \times 10^{-5}$ and $\beta_{n}=1.611 \times 10^{-9}$ [14]. Figure 1 shows the effect of temperature on the effective refractive index in optical fiber. The changes in refractive index are a linear to the increase in temperature.

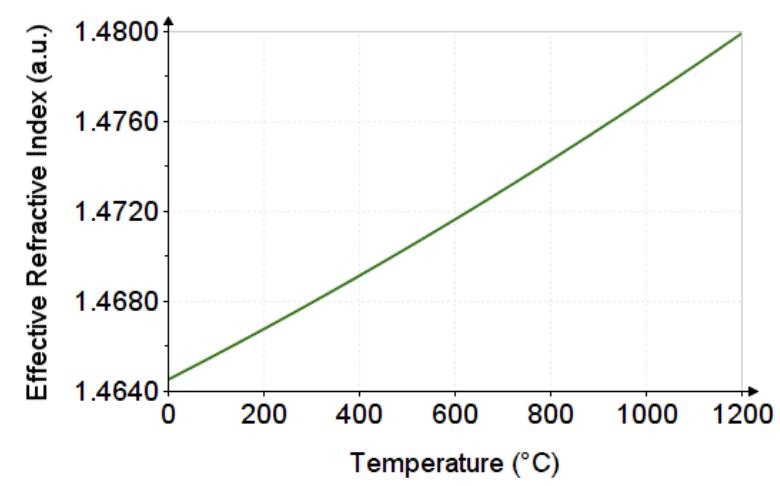

Figure 1. The changes of effective refractive index as temperatures increase

\subsection{Fiber optics Mach-Zehnder interferometers}

The structure of MZI is generally divided into two parts, namely symmetrical MZI and asymmetric MZI [12, 15, 16]. Symmetric MZI has the same length of the upper and lower arm, whereas in MZI the asymmetrical arms have different lengths. In this paper, we just focus on asymmetric MZI. MZI consists of two or more $2 \times 2$ directional couplers which are connected by different lengths in each arm [17-18]. The difference of length made a light wave in one of the optical fiber paths delayed, thus creating a phase shift on the two light beams at the output [19-21]. In this section, MZI consists of two couplers that are connected together on their both ends using optical fiber. This structure is referred to as a single MZI [6, 7]. 
Figure 2 shows the structure of MZI with two couplers, the input signal is launched into port 1 or port 2 and the output can be detected at point 3 and 4 . In this case $L$ is the length of MZI arms and $\Delta L$ is the different length of both arms. The $C_{1}$ is coupling ratio of the first coupler and $C_{2}$ is the coupling ratio of the second coupler. The input light will be divided into two output at first coupler and travels in a different arm length that create a phase sift between the two modes. Further down the two modes will be recombine by coupler 2 and split at the output arms $[9,16]$.

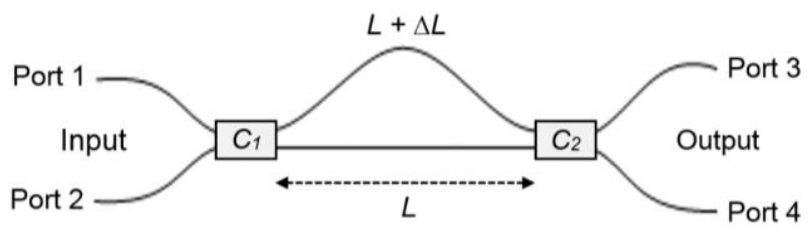

Figure 2. Two couplers MZI diagrams (single MZI)

The output or transmission characteristics of MZI can be explained using the transfer matrix method [16]. It is divided into two main matrices namely couplers and phase shift matrices. The couplers transfer matrix is obtained from [6]:

$$
M_{C}=\left[\begin{array}{ll}
\cos \theta & -j \sin \theta \\
-j \sin \theta & \cos \theta
\end{array}\right]
$$

where $\theta=K L_{c}, K$ is coupling coefficient in the coupler and $L_{c}$ is coupling length in the coupler. This parameter is used to determine the value of the coupling ratio used in the MZI structure [21-23]. Meanwhile, a phase shift matrix can be represented as follows [16, 18]:

$$
M_{\phi_{1}}=\left[\begin{array}{cc}
e^{j \phi_{1}} & 0 \\
0 & e^{-j \phi_{1}}
\end{array}\right]
$$

where [11]:

$$
\phi_{1}=\frac{\beta \cdot \Delta L}{2}
$$

where $\phi_{1}$ is phase shifting on single MZI and $\beta$ is the mode propagation constant in the delay section that determined by:

$$
\beta=\frac{2 \cdot \pi}{\lambda} n_{\text {eff }}
$$

Here $n_{\text {eff }}$ is effective refractive index of optical fiber. Transfer matrixes for single MZI structure are consisting of two transfer matrixes. First is transfer matrixes with coupling coefficient on two couplers and the second is transfer matrix of phase shift $\left(M_{\phi_{1}}\right)$ as shown in Figure 2. So that it can be written as follows:

$$
M_{1}=\left[\begin{array}{cc}
c_{1} & -j s_{1} \\
-j s_{1} & c_{1}
\end{array}\right] \cdot\left[\begin{array}{cc}
e^{j \phi_{1}} & 0 \\
0 & e^{-j \phi_{1}}
\end{array}\right] \cdot\left[\begin{array}{cc}
c_{2} & -j s_{2} \\
-j s_{2} & c_{2}
\end{array}\right]
$$

where, $s_{i}=\sin \theta_{i}=\sqrt{C_{i}}, c_{i}=\cos \theta_{i}=\sqrt{1-C_{i}}$, and $i=1,2,3 \ldots \mathrm{n}$ (number of couplers) and $C_{i}$ is a value of coupling ratio in port 3 . The equation above is the transfer matrix equation for single MZI structure or MZI structure with two couplers [20]. To get an output signal the following calculation might be used as:

$$
\begin{aligned}
& E_{\text {in }}=\left[\begin{array}{l}
E_{\text {in } 1} \\
E_{\text {in } 2}
\end{array}\right] \\
& E_{\text {out }}=\left[\begin{array}{l}
E_{\text {out } 1} \\
E_{\text {out } 2}
\end{array}\right]=\left[\begin{array}{ll}
M_{111} & M_{112} \\
M_{121} & M_{122}
\end{array}\right] \cdot\left[\begin{array}{l}
E_{\text {in } 1} \\
E_{\text {in } 2}
\end{array}\right]
\end{aligned}
$$


The powers outputs for port 3 and port 4 can be obtained by calculate (8) yielding:

$$
P_{\text {out } 1}=\left|E_{\text {out } 1}\right|^{2} \text { and } P_{\text {out } 2}=\left|E_{\text {out } 2}\right|^{2}
$$

Optical Interleaver is a device that can be scaled, so its spectral properties are periodic [9, 12]. The periodicity of the interleaver is referred to as the free spectral range (FSR) i.e. the optical frequency distance or length of two waves of optical intensity that are reflected or transmitted sequentially from an interferometer. Separation of a standard ITU $50 \mathrm{GHz}$ channel requires $\lambda_{0}=1550 \mathrm{~nm}$. The relationship between differences in arm length and FSR is [12]:

$$
F S R=\frac{\lambda_{c}^{2}}{n_{e f f} \Delta L}
$$

where $\lambda_{c}$ is the wavelength center, $n_{\text {eff }}$ is the effective refractive index at that particular wavelength, and $\Delta L$ is the arm different length between two arms of MZI [22, 23]. Hence, (10) might be used to get the length difference needed to obtain a specific FSR at a specific wavelength.

\section{RESULTS AND DISCUSSION}

In this calculation we will analyze several MZI structures that consist of one stage MZI, two-stage cascaded MZI structures, and three-stage cascaded MZI. The output power of each MZI structure are calculated similar to those of single stage MZI except that for each additional cascaded MZI we have to insert a new coupler and phase shift matrix. Furthermore, we also analyze the output characteristics of those MZI structures as a function of temperature changes.

\subsection{Temperature effects on two-stage cascaded MZI}

Figure 3 shows the structure of the two-stages cascaded MZI [9] which consists of three directional couplers and second phase shifters where the second delay line is $2 \Delta L$.

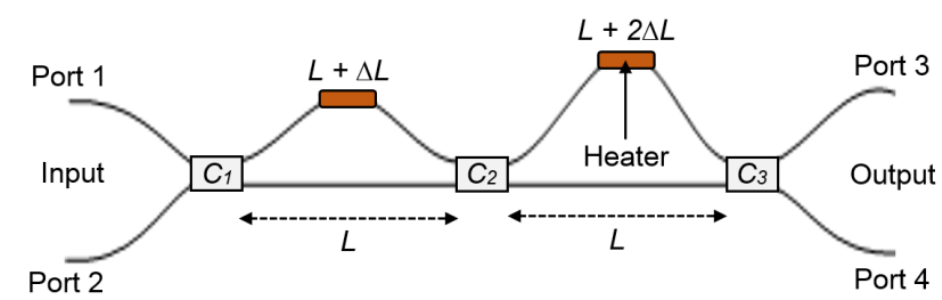

Figure 3. Two-stage cascaded MZI structure with heating element on arms

Therefore, the output field from (4) can be revises to become:

$$
\phi_{2}=\frac{\beta \cdot 2 \Delta L}{2}
$$

Using the similar method as (6) the output power of the two-stage Cascaded MZI is derive as:

$$
M_{2}=\left[\begin{array}{cc}
c_{1} & -j s_{1} \\
-j s_{1} & c_{1}
\end{array}\right] \cdot\left[\begin{array}{cc}
e^{j \phi_{1}} & 0 \\
0 & e^{-j \phi_{1}}
\end{array}\right] \cdot\left[\begin{array}{cc}
c_{2} & -j s_{2} \\
-j s_{2} & c_{2}
\end{array}\right] \cdot\left[\begin{array}{cc}
e^{j \phi_{2}} & 0 \\
0 & e^{-j \phi_{2}}
\end{array}\right] \cdot\left[\begin{array}{cc}
c_{3} & -j s_{3} \\
-j s_{3} & c_{3}
\end{array}\right]
$$

To simulate the power output of the two-stage cascade of the MZI we use the following parameters as shown in Table 1. The coupler coupling ratio is determined based on coupling coefficient and coupling length in $2 \times 2$ optical fiber couplers [16]. To get the spectral response on port 3 and port 4 of two-stage cascade MZI, we have use $(9,12)$. Figure 4 demonstrates the spectral response of two-stages cascaded MZI with the C-Band wavelength spectrum. Spectral responses on the two ports have similar characteristics, where the signal is isolated in the same wavelength range at each particular wavelength. This isolated signal range is called the isolation channel. Separation of channels in each isolation channel is called a crosstalk channel. Directivity or crosstalk is a measure of power reflected from an MZI device. This explains that the 
coupler is very good for transmitting light to the output port and calculating the amount of light reflected in the device [18]. When the crosstalk value is very high it will interfere with propagation. The characteristics of Figure 4 are summarized in Table 2.

One way of changing the silica fiber refractive index is by using the thermo-optical effect. These changes make the loss of transmission low, but still retain its basic features [4]. The temperature effect can also be used as a sensor that has high sensitivity, fast response, and immunity to electromagnetic interference [15]. The effective thermo-optical coefficient depends on the fiber material, temperature changes in each layer, and fiber diameter [14]. The transmission power characteristics of two-stages cascaded MZI as a function of temperature are simulated with a temperature range between $30^{\circ} \mathrm{C}$ (room temperature) to $430^{\circ} \mathrm{C}$ with $\Delta T=100^{\circ} \mathrm{C}$. The output signals are calculated from port 3. Figure 5 shows a shift in the wavelength resulting from changes in temperature in port 3. The simulation results in the $\mathrm{C}$-Band wavelength range and at the center wavelength of $1550 \mathrm{~nm}$. Heating on the Cascaded MZI Two-stage arm only affects the wavelength shift as summarized in Table 2.

Table 1. The simulation parameters

\begin{tabular}{llll}
\hline & Symbol & Unit & Value \\
\hline Parameters & $n_{\text {eff }}$ & & 1.4645 \\
\hline Effective refractive index in fiber & $\lambda$ & $\mathrm{Nm}$ & $1530-1565$ \\
C-Band wavelength range & $a$ & $\mu \mathrm{m}$ & 4.1 \\
Radius of fiber core & $C_{i}$ & $\%$ & $C_{l}=50 / 50$ \\
Coupling ratio of 2×2 optical fiber couplers (port 3/port 4) & & & $C_{2}=75 / 25$ \\
& & & $C_{3}=90 / 10$ \\
& & & $C_{4}=99 / 1$ \\
Arm's length different & $\Delta L$ & $\mu \mathrm{m}$ & 178.3 \\
\hline
\end{tabular}

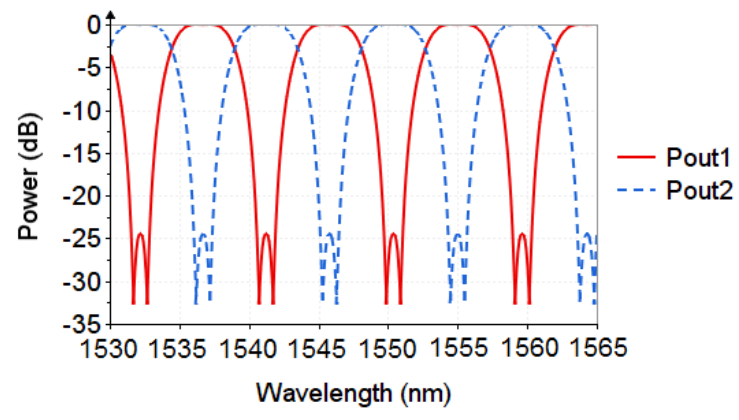

Table 2 Characteristic of two-stage cascaded MZI

\begin{tabular}{ll}
\hline Result & Value \\
\hline Isolation power (port 3 and port 4) & $-32.60 \mathrm{~dB}$ \\
Crosstalk power (port 3 and port 4) & $-24.50 \mathrm{~dB}$ \\
Width of isolation channel & $8.20 \mathrm{~nm}$ \\
Width of crosstalk channel & $1.00 \mathrm{~nm}$ \\
FSR & $9.20 \mathrm{~nm}$ \\
Center wavelength of FSR & $1555 \mathrm{~nm}$ \\
Space between output at port 3 and port 4 & $4.60 \mathrm{~nm}$ \\
\hline
\end{tabular}

Figure 4. Spectral response in port 3 and port 4 of two-stage cascaded MZI

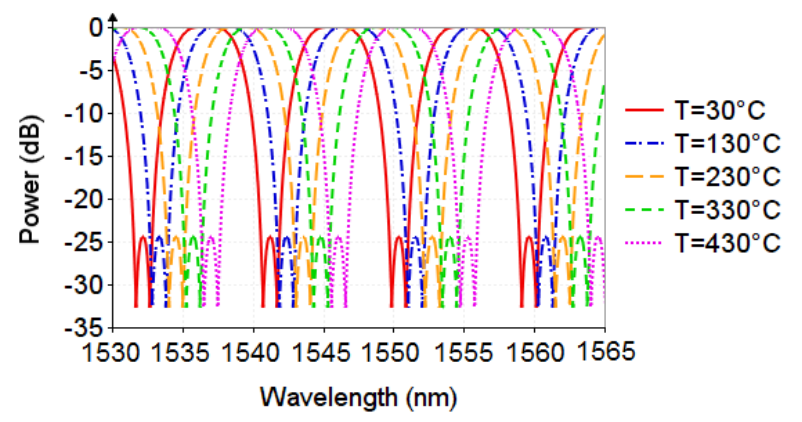

Figure 5. Spectral response of two-stage cascaded MZI (Port 3) with heating on arms

\subsection{Temperature effects on three stages cascaded MZI}

Figure 6 shows the characteristics of the three-stages cascaded MZI which consists of four couplers and three phase shifters where the third delay line is set to be $2 \Delta L$. Similar to the previous analysis, (4) can be revised to become:

$$
\phi_{3}=\frac{\beta \cdot 2 \Delta L}{2}
$$




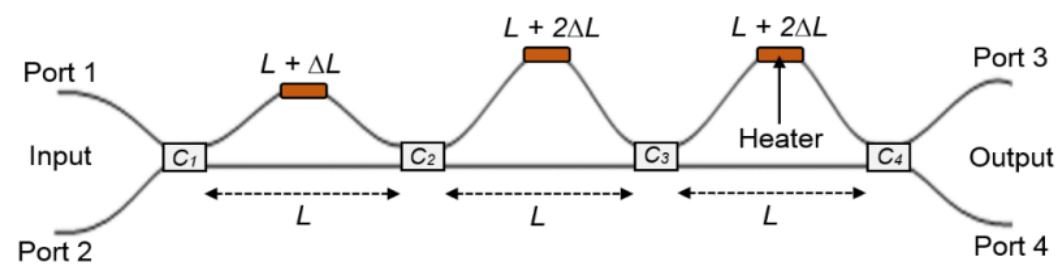

Figure 6. Three-stages cascaded MZI structure with heating element on arms

Using the same method as (6) the output power of the three stages cascaded MZI are derived as follow:

$$
M_{3}=\left[\begin{array}{cc}
c_{1} & -j s_{1} \\
-j s_{1} & c_{1}
\end{array}\right] \cdot\left[\begin{array}{cc}
j \phi_{1} & 0 \\
0 & e^{-j \phi_{1}}
\end{array}\right] \cdot\left[\begin{array}{cc}
c_{2} & -j s_{2} \\
-j s_{2} & c_{2}
\end{array}\right] \cdot\left[\begin{array}{cc}
e^{j \phi_{2}} & 0 \\
0 & e^{-j \phi_{2}}
\end{array}\right] \cdot\left[\begin{array}{cc}
c_{3} & -j s_{3} \\
-j s_{3} & c_{3}
\end{array}\right] \cdot\left[\begin{array}{cc}
e^{j \phi_{3}} & 0 \\
0 & e^{-j \phi_{3}}
\end{array}\right] \cdot\left[\begin{array}{cc}
c_{4} & -j s_{4} \\
-j s_{4} & c_{4}
\end{array}\right]
$$

The spectral response on port 3 and port 4 of two-stage cascade MZI with one input at port 1 is shown in Figure 7. The results from Figure 7 are summarized in Table 3. The spectral response characteristics demonstrate on Table 3 is a bit different from Table 2. The spectral characteristics of the three-stage cascade MZI have the isolation power and the crosstalk power lower than that of two-stage cascaded MZI, but slightly have a loss of $-0.2 \mathrm{~dB}$. Besides that, the width of the crosstalk channel in the schematic design looks wider. Output on both ports has a difference in the isolation power values, additionally the isolation power value at port 4 is higher than that at port 3 . In the same way as in previous section and using parameters in Table 1, the transmission power characteristics of three-stages cascaded MZI as a function of wavelength are simulated using a temperature range $30^{\circ} \mathrm{C}$ to $430^{\circ} \mathrm{C}$ with a range of $\Delta T=100^{\circ} \mathrm{C}$ is demonstrated in Figure 8 . The simulation based on the wavelength range of $1530 \mathrm{~nm}$ to 1565 $\mathrm{nm}$ (C-Band) with the wavelength center at $1550 \mathrm{~nm}$. Value of power isolation, crosstalk power, the width of crosstalk and isolation channel, and loss are similar as those in Table 3. Heating on the cascaded MZI with three-stage arm can only affects the wavelength shift.

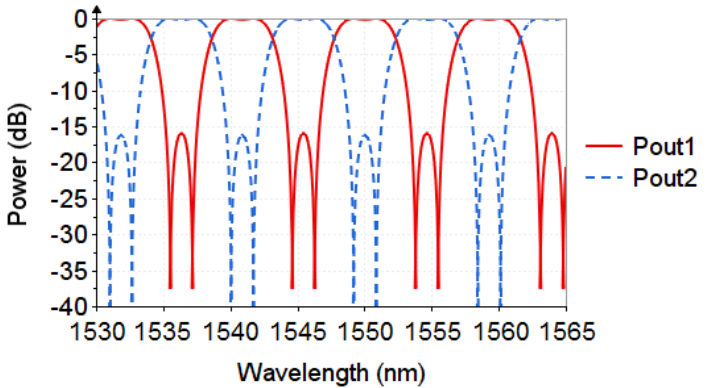

Figure 7. Spectral response in port 3 and port 4 of three-stage cascaded MZI
Table 3. Characteristic of three-stage cascaded MZI

\begin{tabular}{ll}
\hline Result & Value \\
\hline Isolation power in port 3 and port 4 & $-37.5 \mathrm{~dB}$ and $40.0 \mathrm{~dB}$ \\
Crosstalk power in port 3 and port 4 & $-16.0 \mathrm{~dB}$ and $-16.0 \mathrm{~dB}$ \\
Loss & $-0.2 \mathrm{~dB}$ \\
Width of isolation channel & $7.7 \mathrm{~nm}$ \\
Width of crosstalk channel & $1.5 \mathrm{~nm}$ \\
FSR & $9.20 \mathrm{~nm}$ \\
Center wavelength of FSR & $1550 \mathrm{~nm}$ \\
Space between output at port 3 and port 4 & $4.60 \mathrm{~nm}$ \\
\hline
\end{tabular}

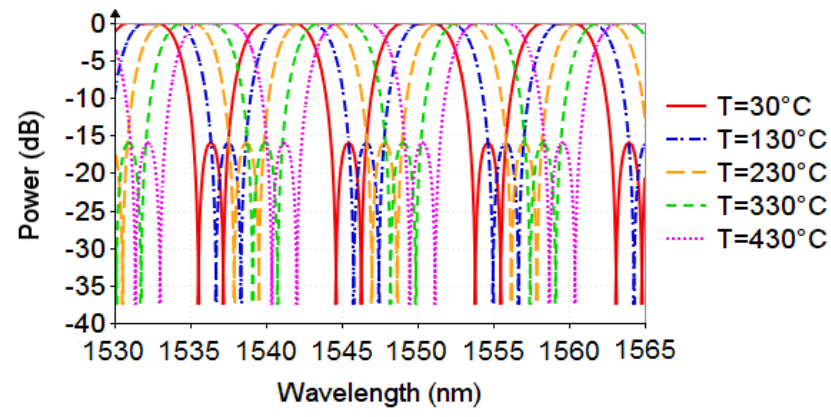

Figure 8. Spectral response of two-stage cascaded MZI at different temperatures 


\subsection{Wavelength shift caused by temperatures}

We have simulated the temperature effect on two-stage and three-stage cascaded MZI in the previous discussion. In this section we focus on the temperature effect of two stages and three-stage cascaded MZI. Based on simulation result in Figures 5 and 7 we obtained that the heating effect on MZI arms that affecting the wavelength shift $[23,24]$. It can be seen that the wavelength shift is increased linearly with the raise of temperatures. Figure 9 shows that the two stages and three-stage cascaded MZI has similar characteristics with the same wavelength shift, therefore we try to find the formulae to get the relation of wavelength shift and temperature changes [25]. From the data in Figure 9, we can find the gradient of graphics as:

$$
m=\frac{4.836}{430-30}=0.01209
$$

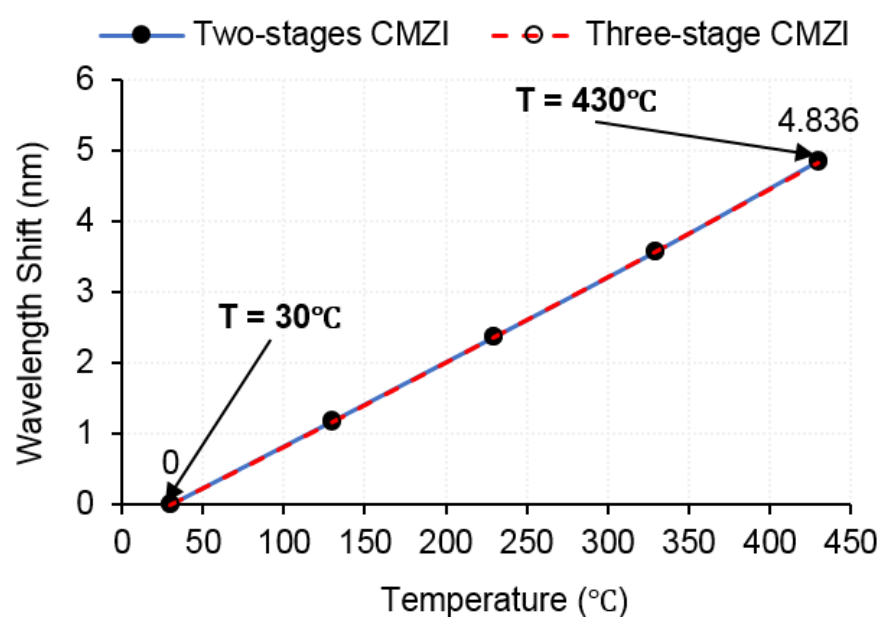

Figure 9. Wavelength shift as a function of temperatures

We can easily obtain the equation between the wavelength shifts due to temperature as [23]:

$$
\Delta \lambda=m T+C
$$

Here $m$ is a gradient, $C$ is a constant. If we substitute (15) into (16), we get:

$$
\left(\lambda_{T^{-}} \lambda_{0}\right)=0.01209\left(T_{T}-T_{0}\right)
$$

where $T_{0}$ and $T_{T}$ are respectively room temperature and final temperature, $\lambda_{0}$ is the wavelength at temperatures $T_{0}$ and $\lambda_{T}$ is the wavelength at the final temperature $\left(T_{T}\right)$. As a result we can easily derive the formula for wavelength shift due temperatures changes as:

$$
\lambda_{T}=\lambda_{0}+0.01209\left(T_{T}-T_{0}\right)
$$

Generally, this equation can be used to predict the wavelength shift against temperature on cascaded MZI. By using the wavelength shift equation, we can immediately choose the right temperature to be used to shift a certain length of wavelengths in cascaded MZI. Further application of this equation it can be used to calibrate the wavelength shift so that it may be used as a sensor device based on optical fiber optics.

\section{CONCLUSION}

It can be concluded that the heating effect in two-stages and in three-stages cascaded MZI cause the wavelength shift. The shape of the cascaded MZI structure does not affect the value of wavelength shift, because the values will be similar on each of structure. Therefore, we can predict wavelength shift by simply using the linear variation of wavelength to the temperature changes as shown in. This equation may be used as a basis of designing the cascaded MZI based thermo-optic effects so that the characteristics of each structure can be predicted in advance. 


\section{REFERENCES}

[1] Stamatios V. Kartalopoulos, "DWDM networks, devices, and technology," John Wiley \& Sons, Inc., Hoboken, New Jersey, 2003.

[2] Disra Agifral, Ary Syahriar, A. H. Lubis, and Sasono Rahardjo, "Simulation of optical switching based on MachZender Interferometer Structure," 2010 The 2nd International Conference on Computer and Automation Engineering (ICCAE), Singapore, pp. 764-767, 2010.

[3] Lu Huaiwei, Wei Yun, Zhang Baoge, Wu Kaijun, and Luo Guanwei, "Study of all-fibers asymmetric interleaver based on Mach-Zehnder interferometer," Optics Communications, vol. 285, no. 6, pp. 1-5, 2012.

[4] R. R. Palupi, A. Syahriar, A. H. Lubis, S. Rahardjo, and Sardjono, "Simulation of Mach Zehnder interleaver based thermo-optic effect in L-Band range," RSM 2013 IEEE Regional Symposium on Micro and Nanoelectronics, Langkawi, pp. 269-272, 2013.

[5] Rekha Mehra, Heena Shahani, and Aslam Khan, "Mach Zehnder interferometer and its applications," International Journal of Computer Applications (IJCA), Proceedings on National Seminar on Recent Advances in Wireless Networks and Communications, pp. 31-36, 2014.

[6] F. A. Ayyubi, A. Syahriar, S. Rahardjo and F. Ali, "Comparison of characteristic of two and three couplers MachZehnder interferometers," 2017 5th International Conference on Cyber and IT Service Management (CITSM), Denpasar, pp. 1-5, 2017.

[7] M. Midrio, M. P. Singh, and C. G. Someda, "The space filling mode of holey fibers: an analytical vectorial solution," Journal of Lightwave Technology, vol. 18, no. 7, pp. 1031-1037, July 2000.

[8] Weibin Li and Junqiang Sun, "Characteristic analysis on an interleaver with a fiber loop resonator by using a signal now graph method," Optica Applicata, vol. XXXVIII, no. 3, pp. 503-510, 2008.

[9] Otto Schwelb, "Cascaded Mach-Zehnder interleaver networks for WDM systems," An Internal Report for OTKA Project T026277, updated April 2005.

[10] A. Harris and P. Castle, "Bend loss measurements on high numerical aperture single-mode fibers as a function of wavelength and bend radius," Journal of Lightwave Technology, vol. 4, no. 1, pp. 34-40, Jan. 1986.

[11] P. Munendhar, L. Zhang, L. Tong, and S. Yu, "Highly sensitive temperature sensor using intrinsic Mach-Zehnder interferometer formed by bent micro-fiber embedded in polymer," 2017 25th Optical Fiber Sensors Conference (OFS), Jeju, pp. 1-4, 2017.

[12] Marek S. Wartak, "Computational photonics," New York: Cambridge University Press. 2013.

[13] J. Buus, "The effective index method and its application to semiconductor lasers," in IEEE Journal of Quantum Electronics, vol. 18, no. 7, pp. 1083-1089, July 1982.

[14] H. Gao, Y. Jiang, Y. Cui, L. Zhang, J. Jia, and L. Jiang, "Investigation on the thermo-optic coefficient of silica fiber within a wide temperature range," Journal of Lightwave Technology, vol. 36, no. 24, pp. 5881-5886, 2018.

[15] J. Hsu, W. Zheng, J. Chen, C. Lee, and J. Horng, "Temperature fiber sensors based on Mach-Zehnder interferometer with sturdy structure," IEEE Sensors Journal, vol. 15, no. 12, pp. 6995-7000, Dec. 2015.

[16] Ary Syahriar, "Mach-Zehnder interferometer for wavelength division multiplexing," Proceedings Komputer dan Sistem Intelijen, Jakarta, pp. A45-A50, 21-22 Agustus 2002.

[17] Md. Haider Ali Shaim and Md. Rezaul Huque Khan, "Design and simulation of a low loss optical fiber coupler," International Journal of Electronics and Communication Engineering, vol. 4, no. 5, pp. 473-482, 2011.

[18] Ci-jun Shuai, Ji-an Duan, and Jue Zhong, "Effect of technological parameters on optical performance of fiber coupler," Journal of Central South University Technology, vol. 14, vol. 3, pp. 370-373, 2007.

[19] Ali Reza Bahrampour, Sara Tofighi, Marzieh Bathaee, and Farnaz Farman, "Optical fiber interferometers and their applications," In Interferometry-Research and Applications in Science and Technology, InTech, pp. 3-30, 2012.

[20] A. Kurysheva, "All-fiber Mach-Zehnder interferometer for DWDM-PON bidirectional multiplexing," M.S. Thesis, Departament de Teoria del Senyal i Comunicacions, Universitat Politècnica de Catalunya, Barcelona, Spain, 2015.

[21] Huai-Wei Lu, Kai-jun Wu, Yun Wei, Bao-Ge Zhang, and Guan-Wei Luo, "Study of all-fiber assymmetric interleaver based on two stage cascaded Mach Zehnder interferometer," Journal Optical Communication, vol. 285, no. 6, pp. 1118-1122, 2012.

[22] Francesca Magno, Francesco Dell'Olio, and Vittorio M. N. Passaro, "Multiphysics investigating of thermo-optic effect in silicon-on-insulator waveguide arrays," Proceeding of the COMSOL Users Conference, Milano, 2006.

[23] Maria L. Calvo and Vasudevan Lakshminarayanan, "Optical waveguide from theory to the applied technology," CRC Press, New York, 2007.

[24] C. Galland, A. Novack, Y. Liu, R. Ding, M. Gould, T. Baehr-Jones, Q. Li, Y. Yang, Y. Ma, Y. Zhan, and K. Padmaraju, "A CMOS-compatible silicon photonic platform for high-speed integrated opto-electronics," Proceedings Integrated Photonics: Materials, Devices, and Applications II; vol. 8767, p. 87670G, May 2013.

[25] Ye Tian, T. Tan, C. Duan, B. Xu, X. Zhao, Q. Chai, J. Ren, J. zhang, E. Lewis, Y. Liu, and J. Yang, "High sensitivity liquid level sensor based on dual side-hole fiber Mach-Zehnder interferometer," Optics Communications, vol. 440, pp. 194-200, June 2019. 


\section{BIOGRAPHIES OF AUTHORS}
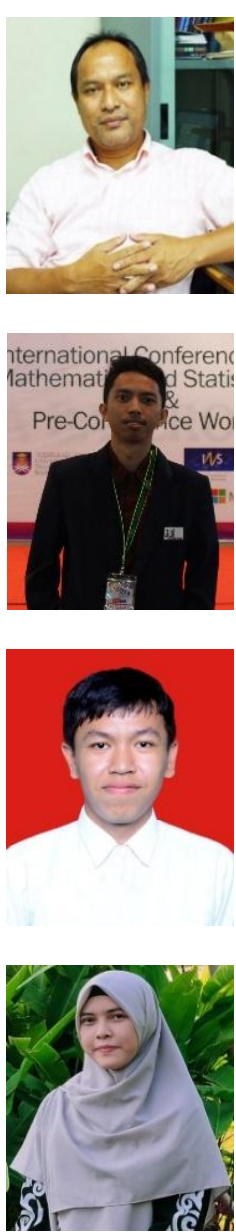

Putri Wulandari received her MSc degree in Computer Science from Universiti Brunei Darussalam - Brunei Darussalam in September 2016. She received her Bachelor's degree in Electrical Engineering from Universitas Al Azhar Indonesia in 2012. She is currently a Lecturer in the Department of Electrical Engineering at Universitas Al Azhar Indonesia. Her research interests include Renewable Energy, Telecommunication Satellite, Biomedical Engineering and Antenna. 